\title{
The Design and Construction of a High Endurance Hexacopter suited for Narrow Corridors*
}

\author{
J. Verbeke, D. Hulens, H. Ramon, T. Goedemé and J. De Schutter
}

\begin{abstract}
We have conceived a novel compound multicopter (helicopter type utilizing multiple different size propellers for separate lift and attitude control) configuration specifically for flight through narrow corridors. Its design combines the contradictory requirements of limited width, high agility and long endurance while carrying a significant payload. This configuration can be scaled for both indoor and outdoor applications. The development is part of a doctoral research in which an autonomous unmanned rotary helicopter is designed, constructed and flight tested for inspecting fruit orchards and vineyards while flying in between the tree rows in outdoor conditions such as wind and gusts. The compound hexacopter configuration combines two large lift propellers, with a constant rotational velocity, with four small control propellers commanded by an autopilot. The autopilot is configured as a quadcopter commanding only the control propellers as only these change the attitude and overall thrust of the hexacopter. The benefit of using large lift propellers is their lower disk loading (thrust divided by disk area) which results in a higher Figure of Merit and lower power consumption compared to the smaller control propellers, while the latter are better suited for outdoor (windy) conditions due to their fast reaction time in spooling up and down. Compared to a standard quadcopter with the same width, payload and battery capacity, the endurance of the compound hexacopter is potentially up to $60 \%$ higher. As a concept validator, a small-scale prototype has been designed, constructed and successfully flight tested.
\end{abstract}

\section{INTRODUCTION}

A lot of research is performed on autonomous control of unmanned aerial vehicles (UAVs). UAVs come in all sizes, fixed wing or rotary, and are used to perform various applications [1], largely in the field of remote sensing [2]. Currently, there is a growing interest in using rotary UAVs for applications in confined spaces, more specifically in corridors, both indoor and outdoor. Potential applications include searching collapsed buildings [3], searching criminals in houses by the Police [4] and inspecting fruit orchards and vineyards from in between the tree rows [5]. The main challenges of rotary UAV for flying through corridors are the limited width and the required agility, especially in outdoor conditions where wind and gusts cause the UAV to drift.

* Research supported by Kulab (Propolis) and KULeuven (FWO-project 6.0404.10).

$\mathrm{J}$. Verbeke is with the Department of Mechanical Engineering PMA, KU Leuven, Leuven, 3001 Belgium (e-mail: jon.verbeke@kuleuven.be).

D. Hulens is with the Department of Electrical Engineering ESAT, KU Leuven, Leuven, 3001 Belgium (e-mail: dries.hulens@thomasmore.be).

J. De Schutter is with the Department of Mechanical Engineering PMA, KU Leuven, Leuven, 3001 Belgium (e-mail: joris.deschutter@mech.kuleuven.be).

T. Goedemé is with the Department of Electrical Engineering ESAT, KU Leuven, Leuven, 3001 Belgium (e-mail: toon.goedeme@esat.kuleuven.be).

H. Ramon is with the Department of Biosystems BIOSYST, KU Leuven, Leuven, 3001 Belgium (e-mail: herman.ramon@biw.kuleuven.be).
Compared to a conventional (main and tail rotor) helicopter, a multicopter is more suited for flights in confined spaces. A multicopter is a type of helicopter that has more than two rotors/propellers that lift and control the platform (Fig. 1). Most multicopter platforms utilize fixed-pitch propellers that control the platform by changing their rotational velocities. The number of propellers is unlimited. The main advantages of a multicopter compared to a conventional helicopter are its inherent high agility and compactness which, for flying through corridors, are of utmost importance. The high agility is required for quick response to sudden external disturbances such as gusts of wind in order to minimize the drift while flying through the corridor. The compactness is essential as corridors are often narrow. The main disadvantage of using a multicopter for flights through corridors is that even though it is already compact, the width restriction forces the use of small propellers compared to the weight they have to carry. This results in a lower Figure of Merit and thus short endurance (20min or less for a typical quadcopter smaller than $1 \mathrm{~m}$ such as the Pelican AscTec shown in Fig. 1).

We conceived a novel multicopter configuration to deal with these contradictions. It is a high endurance compound asymmetrical hexacopter specifically suited for flight through narrow corridors. The combination of an asymmetrical compound configuration and innovative method of control has not yet been proposed. The compound hexacopter configuration can be used for both indoor and outdoor applications and can be scaled to the preferred size. The Demonstrator, a small-scale prototype without the payload, enables validating the novel configuration and its performance during flight.

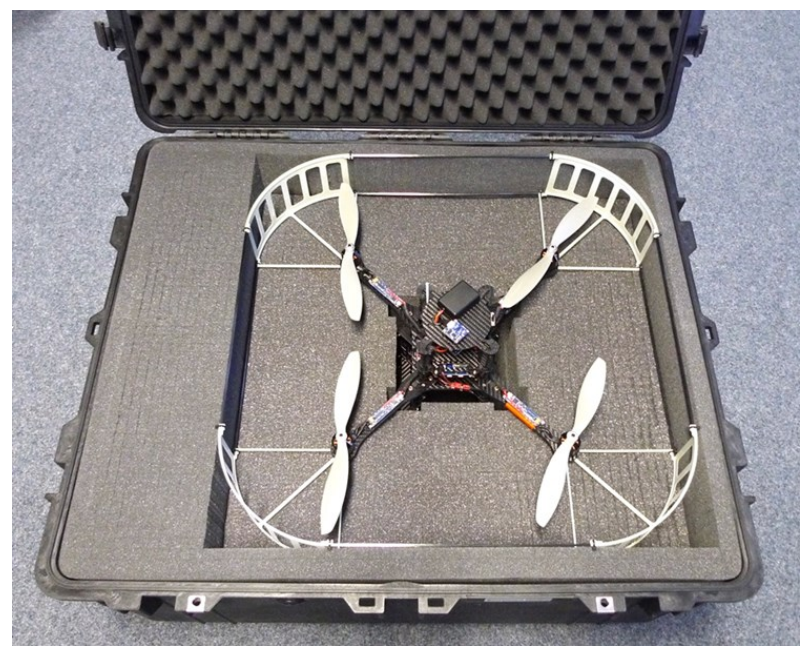

Figure 1. A typical quadcopter (Pelican AscTec) 
Our research focusses on the design, construction and flight testing of a rotary UAV for inspection of orchards and vineyards. One of the innovating aspects is that the unmanned helicopter will autonomously fly in between tree rows and use sideward looking cameras for inspection instead of flying over the orchard like other UAVs do [6][7]. Flying in between tree rows is very similar to flying through a corridor and for this reason a suitable platform was conceived resulting in the compound hexacopter. The fruit orchard is assumed to be of the vertical axe system or fruiting wall type, where branches are not sticking out into the corridor (Fig. 2). The main application would be harvest yield estimation. As weather has a great influence on the growth of fruit, large fluctuations occur from year to year and even from orchard to orchard. Currently, farmers sample a certain percentage of the orchard and extrapolate these measurements to the entire vineyard. This usually disregards the size of the fruit and is only done once during the entire growing season. As a result, yield estimations can be off by up to $20 \%$ [8]. Knowing the yield, even at an early stage during the growing season, has great advantage for the farmer as he can adjust his cultivation techniques accordingly. For instance, early in the growing season, flower and fruit thinning [9] are used to improve fruit size and reduce alternate bearing in apple trees. Accurate yield estimation and thinning techniques require automation in order to be feasible and commercially viable for large orchards and vineyards. Sideward looking cameras perform the fruit yield measurements by measuring the size of each fruit and estimating the total fruit weight in the orchard. Accurate yield estimation requires high spatial accuracy in the order of millimeters. Using overflying UAVs to achieve this level of accuracy requires very expensive cameras with an extremely high resolution and a narrow field of view lens. A much more economical solution is to fly very close to the fruit. For example, a UAV flying at $50 \mathrm{~m}$ above the orchard with a very narrow field of view $\left(10^{\circ}\right)$ requires a $22 \mathrm{MP}$ camera to achieve $6 \mathrm{~mm}$ spatial accuracy while a $0.5 \mathrm{MP}$ camera with a wide field of view $\left(100^{\circ}\right)$ at a distance of $1 \mathrm{~m}$ already results in a spatial accuracy of $3 \mathrm{~mm}$. Close-up inspection requires a much lower quality camera and image processing power thus allowing onboard real-time processing of the images while the weight of the payload remains relatively low. Early biotic stress detection is another inspection that requires high spatial accuracy as the first symptoms appear at leaf level [10]. As a final note, the closeup inspection method minimizes the effects of soil background noise, hail nets, leaf drooping and shadow with respect to data quality.

Naturally, one has to ask the question: why go with a UAV instead of a ground-based robot that could drive through the orchard as this will have fewer problems with carrying heavier payload and computer hardware? However, a UAV is not affected by the terrain (hills, slopes, potholes, muddy terrain during rainy seasons...). For example, to inspect the higher region of the fruit trees, the camera would have to be attached to a pole a few meters above the ground vehicle. This requires a system that counters the tilting of the ground robot caused by terrain roughness to keep the camera stable (and out of the trees). Another option would be to stop in front of every tree which is very time-consuming. In addition, if the design of the sideward looking camera setup of the UAV is such that it allows for easy adaptation towards an overflying, downward looking pose, then even larger areas can be inspected (albeit with lower spatial accuracies) in a much shorter time frame. The main disadvantage of using a multicopter UAV is its short endurance which the novel compound design presented in this paper improves significantly.

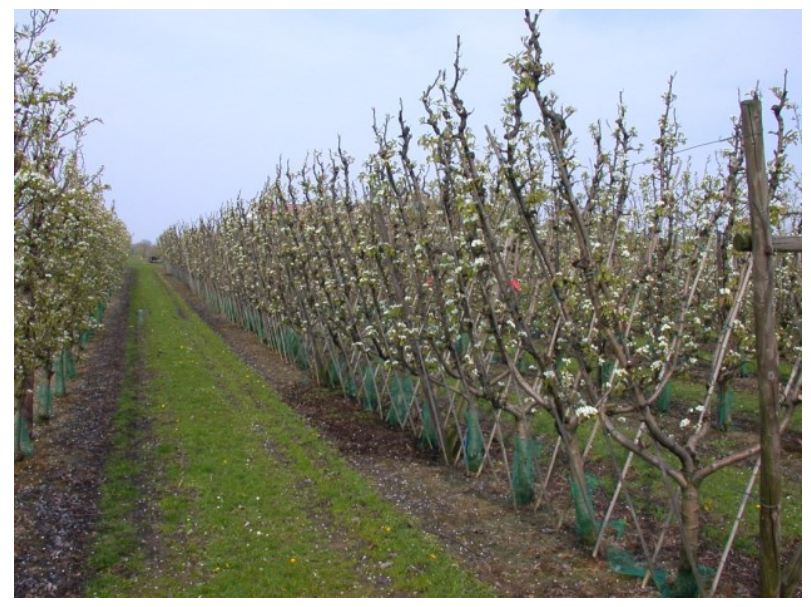

Figure 2. The corridor inbetween fruit tree rows

This paper describes the design and control of the new compound configuration and compares its endurance to a standard multicopter. Furthermore, the design, construction and initial flight tests of a small-scale prototype, named the Demonstrator, specifically designed for validating the compound configuration's control and performance are discussed.

\section{THE COMPOUND HEXACOPTER CONFIGURATION: MECHANICAL DESIGN}

Flying a multicopter through a corridor requires a minimal available working width as a safety margin is needed to allow small drift from the multicopter due to external disturbances. For the harvest yield estimation UAV, a $2 \mathrm{~m}$ wide corridor is assumed with on either side $0.5 \mathrm{~m}$ margin for drift, leaving $1 \mathrm{~m}$ for the width of the multicopter. To be commercially viable, the multicopter is required to have a high endurance to be able to inspect large areas. High endurance means large battery capacity and thus high battery weight. In addition, the payload and computer hardware also add significantly to the total weight. Due to the width restriction, a standard multicopter would have small propellers (short radius $\mathrm{R}$ ) compared to the weight $\mathrm{W}$ they carry, in other words a high disk loading DL (1) [11].

$$
D L=\frac{W}{\pi R^{2}}=\left[\frac{N}{m^{2}}\right]
$$

Yet, during hover and slow forward flight, where thrust $\mathrm{T}$ (approximately) equals weight $\mathrm{W}$ (2), a low disk loading and thus low induced propeller wash velocity $\mathrm{v}_{\mathrm{i}}$ is preferred (3) in order to efficiently generate thrust $\mathrm{T}$, keep the required power $\mathrm{P}_{\text {hov }}$ low (4) and have a long endurance.

$$
\begin{aligned}
T_{h o v} & =2 \dot{m} v_{i_{h o v}}=2 \rho \pi R^{2} v_{i_{h o v}}^{2}=W \\
& \Rightarrow v_{i_{h o v}}=\sqrt{\frac{W}{2 \rho \pi R^{2}}}=\sqrt{\frac{D L}{2 \rho}}
\end{aligned}
$$




$$
\Rightarrow P_{\text {hov }}=T_{\text {hov }} v_{i_{\text {hov }}}=W v_{i_{\text {hov }}}=W \sqrt{\frac{D L}{2 \rho}}
$$

The downside of using a large propeller is that its high inertia requires not only large power inputs to change its rotational velocity, but foremost reaction time. When the helicopter is flying in choppy winds, this delay in response can be devastating for the platform's performance, especially when flying through narrow corridors where the drift has to be kept minimal. Therefore, high agility requires small propellers.

The compound hexacopter has a rectangular shape and combines large lift and small control propellers to achieve high endurance (Fig. 3). The rectangular shape expands the limited space needed for generating lift. Two large lift propellers $\left(\mathrm{L}_{1}\right.$ and $\left.\mathrm{L}_{2}\right)$ are combined with four small control propellers $\left(\mathrm{C}_{1}, \mathrm{C}_{2}, \mathrm{C}_{3}\right.$ and $\left.\mathrm{C}_{4}\right)$ around a central payload box. In addition to the configuration, the method of control is also an innovative feature: the lift propellers are counter-rotating $\left(\omega_{\mathrm{L} 1}\right.$ and $\left.\omega_{\mathrm{L} 2}\right)$ at a constant speed to deliver most of the lift required in hover, but not all. The control propellers are commanded by the autopilot, which is actually configured to behave as a quadcopter with an adapted flight model. The lift propellers rotating at high rotational velocity act as gyroscopes when the multicopter performs maneuvers resulting in unwanted roll/pitch moments that need to be accounted for in the flight model. In addition, when the multicopter is tilted, the thrust vector of the lift propellers tilts as well, lowering the vertical thrust component. By accounting for these effects in the flight model of the quadcopter used for attitude control, the unwanted lift propeller effects can be minimized when combined with a suitable controller.

Since the lift propellers only need to deliver a constant thrust, the power margin (ratio of installed power w.r.t. hover power) can be much smaller as well as the motors, saving weight, compared to standard hexacopters. Note that without width restriction, a standard multicopter with large propellers and hence low disk loading may yield the same endurance as the compound UAV. For instance, the MD4-1000 can achieve a flight time of more than one hour thanks to its large propellers compared to its low weight resulting in a very low disk loading [12]. The compound hexacopter configuration presented here is specifically intended for narrow corridor flights. The harvest yield estimation compound hexacopter, with its length of two meter and width of one meter, would be one of the largest multicopters in existence, apart from the e-volo VC1 [13] and MD4-3000 [14].

There are some aspects of this configuration that need to be addressed. First of all, the central payload box configuration, with the control propellers close-by and the lift propellers outboard, is chosen to minimize the moment of inertia of the hexacopter (Fig. 10). The closer the heavy objects are to the center of gravity the less moment the control propeller pairs have to generate. The position of the control propellers together with the asymmetrical inertia of the compound design results in the UAV being less responsive in pitch than in roll. However, for the use in a narrow corridor, the lateral drift due to external disturbances is of higher importance since the trees are only on the side of the UAV.

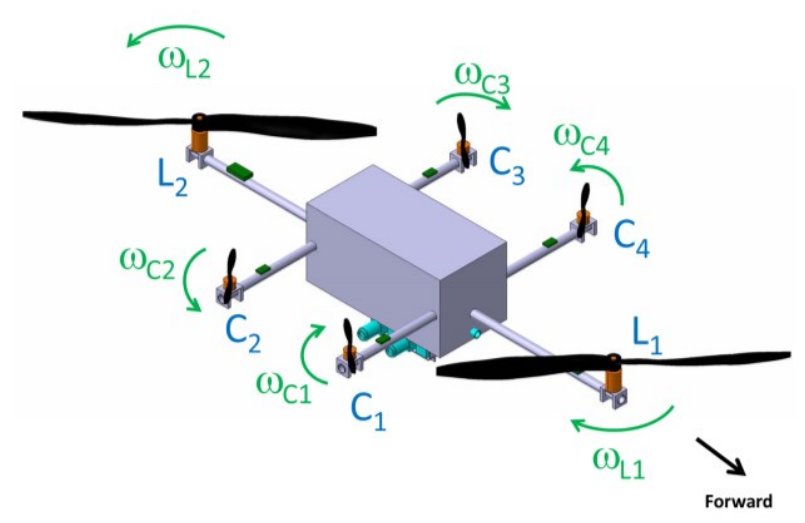

Figure 3. The novel compound hexacopter configuration

An alternative to this could be to move the pitch control propellers fore and aft of the UAV, but this would require a heavier structure to keep the stiffness of the UAV high as is needed for fast and accurate attitude response. Furthermore, the UAV would be less compact which is crucial for indoor operations. This alternative configuration could be a solution if the pitch control of the current configuration proves insufficient for outdoor operations, which will be tested in the near-future with the prototype. Another concept, such as central counter-rotating lift propellers with control propellers around it, would require the payload to be distributed around the central propellers thus significantly adding moment of inertia and is therefore considered unsuitable. H-shaped multicopters have been designed with lengths longer than the widths that are rather well suited for flying through corridors. However, they still use many small propellers which results in a short endurance [15].

\section{THE COMPOUND HEXACOPTER CONFIGURATION: CONTROL AND HARVEST YIELD APPLICATION DESIGN}

Apart from the narrow corridor design, the combination of large lift propellers with small control propellers is a very good solution for the design limitations of large multicopter UAVs. The main problem with scaling up multicopters is that for control it relies on rapidly changing the thrust of each motor-propeller combination which is mostly done by changing the rotational velocity. If the propeller gets larger, the higher inertia diminishes the capacity of rapidly spooling down the propeller (as the brushless DC motors cannot brake) which means there is a delay to the controller outputs. Currently, research and industry solve this by replacing the propeller by a rotor with pitch control [16] or many small propellers [13]. The complex pitch control mechanism significantly increases the possibility of failure. A better solution would be to use a central payload, add large lift propellers (with constant rotational velocity) around it and then use small control propellers outboard of or in between the lift propellers. The compound configuration would be much better suited for outdoor conditions as it keeps the weight low and the robustness intact, one of the main advantages of multicopters. In addition, it opens up new perspectives for hybrid multicopters where the lift propellers are directly powered by a central gasoline driven motor, which is more efficient than adding an onboard generator with its own power losses. 
To make this configuration successful, a suitable controller is required. Although the stabilization and navigation methods described here are for navigating safely through corridors in between trees, the method is generic enough to be adapted to all sorts of corridors. The flight controller (FC) collects data from an Inertial Measurement Unit (IMU), a GPS, a barometric pressure sensor (absolute altitude) and two ultrasonic altitude sensors (for relative altitude above ground) to navigate outside of the corridors (Fig. 11). The IMU estimates the attitude very accurately, but the derived velocities and positions show a large drift over time. The GPS achieves reasonable accuracy (less than one meter) and is used for absolute position determination and drift correction outside the tree rows. However, in between the tree rows a forward looking camera is used for drift correction as the accuracy of the UAV's position has to be in the order of centimeters. Once positioned in front of the tree row corridor, the forward camera image processing becomes dominant. The UAV has two Image Processing Units (IPUs) onboard, mainly for processing the payload camera images (two cameras per IPU), but also for processing the forward camera images.

The IPU performs real-time image processing on the forward camera images based on color and texture segmentation to find the ground, trees and sky. Subsequently, custom software estimates the relative position of the quadcopter with respect to the middle of the corridor. In addition, the software determines the middle of the corridor at the horizon to make trajectory estimations and uses it to keep the nose pointed to the end of the corridor and the payload cameras aimed at the fruit trees. The software has already been implemented on a Parrot AR Drone and flight tested in a fruit orchard with promising results, as described in [17]. The position estimation and trajectory generated by the IPU are sent to the FC and combined with the data from the IMU, GPS, ultrasonic sensors and barometric pressure sensor to provide accurate current and future desired position and velocities of the UAV. The flight controller then calculates the required power settings and commands the electromotors driving the control propellers. In the nearfuture, ultrasonic sensors on each side of the UAV may be added, as a backup, to help avoiding the "wall of fruit trees", while the forward camera image processing software can be expanded with object detection algorithms for avoiding possible stray branches extending into the corridor. Currently, these added safety features are not included in the design but can be added in the future. Both data from the IPU and the FC are sent wirelessly to the ground station by the telemetry unit.

Table III presents the provisional list of all components of the full-scale compound UAV used for the conceptual design and performance calculations. Four monochrome fruit inspection cameras are used in pairs to inspect both sides of the tree corridor simultaneously (Fig. 4). They are in a stereo setup on a 1D gimbal to, based on comparing the photos of both cameras, calculate the distance to each fruit and derive the size (and weight) of the fruit. For now, the gimbal only counters the rolling motion of the multicopter (due to weight considerations), but if required the swing can be easily upgraded to a $2 \mathrm{D}$ version. Each cameras is $0.5 \mathrm{MP}$, has a lens with a wide $\left(104^{\circ} \times 77^{\circ}\right)$ angle of view and a special wavelength bandpass filter that improves the contrast between fruit and tree (leaves). This setup ensures a spatial accuracy of $3 \mathrm{~mm}$ and a maximum forward velocity of the UAV of $7 \mathrm{~km} / \mathrm{h}$ based on a $25 \%$ image overlap and two frames per second image processing power of the IPU. With this setup, for a tree row length of $150 \mathrm{~m}$ and $36 \mathrm{~min}$ flight time, approximately $15000 \mathrm{~m}^{2}$ could be inspected or around 2100 fruit trees. For the forward camera a low-resolution camera $(720 \times 480 p)$ is selected as this is sufficient for trajectory planning.

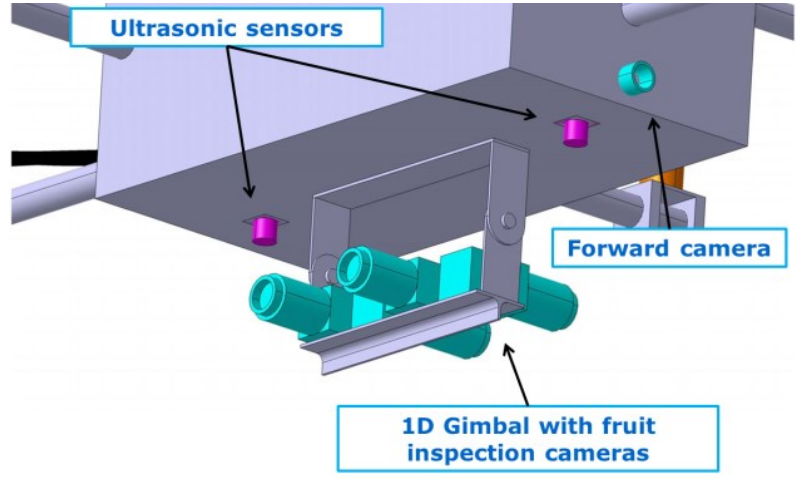

Figure 4. The fruit inspection cameras with 1D gimbal

\section{PERFORMANCE COMPARISON WITH A STANDARD MULTICOPTER}

Basic momentum disk theory has been used to estimate the performance of the novel configuration [18]. The estimations could be improved by using the blade element method, but suffice for this basic comparison. This performance was compared to a standard quadcopter with the same width and payload (Fig. 5) (Table IV) to prove the higher endurance of the compound design. A quadcopter was chosen over a hexacopter configuration as a quadcopter has a larger total propeller area than a hexacopter when its width is limited. The quadcopter's design is made similar to the Demonstrator's design with a maximum width of one meter. The components are custom selected and the propeller size is maximized. For basic comparison the structural weight, payload weight and computer hardware weight were chosen equal.

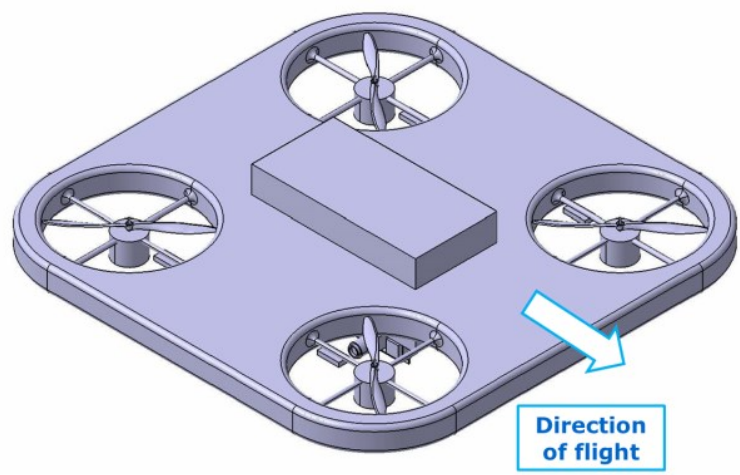

Figure 5. The standard quadcopter used for comparison

The comparison (Table I) shows the compound UAV has potentially up to $60 \%$ more endurance than the quadcopter, mainly due to the difference in disk loading and hence propulsion efficiency. Keep in mind that cheap non- 
optimized propulsion components are used in the initial design which means that a design using high-end components will increase the $36 \mathrm{~min}$ flight time of the compound design dramatically. Based on this high endurance potential, a smallscale version ( $60 \%$ size of full-scale) of the compound UAV without the harvest yield estimation payload was designed and constructed, named the Demonstrator. The purpose of the Demonstrator is to validate the novel multicopter configuration and its performance.

\section{THE DEMONSTRATOR UAV}

The Demonstrator has an endurance of $12 \mathrm{~min}$, well suited for (indoor) flight testing (Table II). Note that the limited flight time is due to the use of low-end, non-optimized components. With high-end custom components, the flight time would be significantly higher. Keep in mind that the Demonstrator is intended solely as a concept validator, a roll it fulfills very well. The efficiency of the propellers and motors, power margin, power consumption vs. thrust and battery effective capacity are determined by motor-propeller tests (Fig. 6) and used in the performance calculations so as to precisely determine the endurance and battery capacity required. Flight tests validated these parameters. In addition, tests determined the optimal combinations of motor, Electronic Speed Controller (ESC) and propeller for the Demonstrator based on electrical power consumption and response. Compared to a similar size standard quadcopter with four $25.4 \mathrm{~cm}$ diameter (10inch) propellers the validated endurance is $25 \%$ higher, compared to the theoretical $60 \%$ of the conceptual full-size compound hexacopter.

TABLE I. PERFORMANCE COMPARISON BETWEEN THE COMPOUND UAV AND STANDARD QUADCOPTER

\begin{tabular}{|c|c|c|c|}
\hline Parameter & Quad & Compound & Units \\
\hline \multirow{2}{*}{$\begin{array}{l}\text { \# propellers } \mathrm{x} \text { propeller } \\
\text { diameter }\end{array}$} & \multirow{2}{*}{$\begin{array}{r}4 \times 30.5 \\
(4 \times 12 \text { inch })\end{array}$} & $\begin{array}{r}2 \times 76.2 \\
(2 \times 30 \text { inch })\end{array}$ & $\mathrm{cm}$ \\
\hline & & $\begin{array}{r}4 \times 20.3 \\
(4 \times 8 \text { inch }) \\
\end{array}$ & $\mathrm{cm}$ \\
\hline Disk loading & 224 & 64 & $\mathrm{~N} / \mathrm{m}^{2}$ \\
\hline \multirow{2}{*}{ Figure of Merit } & \multirow{2}{*}{70} & Lift 75 & \multirow{2}{*}{$\%$} \\
\hline & & Control 70 & \\
\hline Motor + ESC efficiency & 80 & 80 & $\%$ \\
\hline \multirow{2}{*}{$\begin{array}{l}\text { Power required per motor } \\
\text { (hover) }\end{array}$} & \multirow[t]{2}{*}{279} & 216 & Watt \\
\hline & & 38 & Watt \\
\hline Payload energy required & 66 & 66 & Watt \\
\hline $\begin{array}{l}\text { Total power required } \\
\text { (hover) }\end{array}$ & 1182 & 650 & Watt \\
\hline Mass payload & 0.70 & 0.70 & $\mathrm{~kg}$ \\
\hline Mass computer hardware & 0.49 & 0.49 & $\mathrm{~kg}$ \\
\hline Mass propulsion & 4.47 & 4.38 & $\mathrm{~kg}$ \\
\hline Mass structure & 1.00 & 1.20 & $\mathrm{~kg}$ \\
\hline Total mass UAV & 6.66 & 6.77 & $\mathrm{~kg}$ \\
\hline Endurance & 23 & 36 & Min \\
\hline Endurance improvement & & 58 & $\%$ \\
\hline
\end{tabular}

The Demonstrator has some dissimilar design features compared to the conceptual design of the full-scale UAV (Fig. 7 and Fig. 8). First of all, the structure is a commercialof-the-shelf modified glass fiber hexacopter frame. Secondly, we designed the electrical system such that the control and lift propulsion is separated as much as possible for safety reasons including separate batteries and monitoring devices.
The brushless DC motors are commanded by an open-source ArduPilot-Mega (APM) 2.5 autopilot [19] through ESCs. The autopilot is based on Proportional-Integrative-Derivative (PID) architecture. A 3DR radio telemetry system, which is a type of open source Xbee radio, is used by the APM to communicate with a laptop serving as ground station through the Mission Planner software, that comes with the ArduPilot autopilot. The lift propulsion motors are directly commanded through the receiver by a rotary knob on the pilot's transmitter as they do not have to help with pitch control, but need to deliver a constant thrust. This does have a negative side-effect due to characteristics of the Lithium Polymer batteries. In the fully charged state, each cell generates $4.2 \mathrm{~V}$, yet as it discharges this drops to $3.2 \mathrm{~V}$. As a result, delivering a constant power to the motor requires increasing the current accordingly. The current ESC is not capable of correcting for this. During flight tests the correct transmitter setting based on the battery's charge state is estimated and set into the transmitter to keep the lift propulsion setting around $75 \%$ of the total weight as envisioned. This will be improved in the near-future by using an ESC with governor option. This is used in conventional helicopters and keeps the rotational speed of the propeller constant despite the voltage drop over time. In addition, the electrical system will be upgraded to improve the onboard safety monitoring and power management.

TABLE II. THE SPECIFICATIONS OF THE DEMONSTRATOR UAV

\begin{tabular}{|c|c|c|}
\hline Parameter & Value & Unit \\
\hline Computer harware mass & 0.356 & $\mathrm{~kg}$ \\
\hline Propulsion mass & 1.171 & $\mathrm{~kg}$ \\
\hline Structural mass & 1.194 & $\mathrm{~kg}$ \\
\hline Total mass & 2.720 & $\mathrm{~kg}$ \\
\hline Lift prop diameter & $\begin{array}{r}40.6 \\
\text { (16inch) }\end{array}$ & $\mathrm{cm}$ \\
\hline Control prop diameter & $\begin{array}{r}20.3 \\
\text { (8inch) }\end{array}$ & $\mathrm{cm}$ \\
\hline Overall length & 1.231 & $\mathrm{~m}$ \\
\hline Overall width & 0.690 & $\mathrm{~m}$ \\
\hline Overall height & 0.340 & $\mathrm{~m}$ \\
\hline Figure of Merit & 60 & $\%$ \\
\hline Motor + ESC efficiency & 75 & $\%$ \\
\hline Endurance & 12 & $\min$ \\
\hline Hover thrust Lift/Control & $75 / 25$ & $\%$ \\
\hline Power margin Lift & 1.21 & - \\
\hline Power margin Control & 2.18 & - \\
\hline Battery type & $3 \mathrm{~S}$ & LiPo \\
\hline Total battery capacity & 8700 & $\mathrm{mAh}$ \\
\hline
\end{tabular}

The third altered design feature is the difference in propeller disk loading as extra weight was added due to an extension of the landing gear to prevent it from tilting over on the ground and protect the propellers. This resulted in a higher disk loading of the lift propellers. Additionally, the small control propellers' Figure of Merit was lower than expected and they were upgraded from $12.1 \mathrm{~cm}$ (4.75inch) diameter to $20.3 \mathrm{~cm}$ (8inch), resulting in a lower disk loading, despite the increased total weight. The propellers can be easily substituted by other ones and the disk loading ratio between the lift and control propulsion parameter will be 
experimented with in the future. Furthermore, the ratio of thrust that lift and control propellers generate in hover is $75 / 25 \%$ respectively, while for the full-scale this was assumed at $80 / 20 \%$. There is margin to experiment with this setting as it may be wise to increase the control margin in gusty conditions. A better suited landing gear is currently being conceived. As a last feature, the control propellers are tilted by $10^{\circ}$ to improve the yaw control of the Demonstrator as the first flight test showed inadequate response. Do note that the APM simply "thinks" it's a quadcopter with high and asymmetrical inertias which are overcome by using different controller settings for pitch and roll. The APM currently does not use an extended quadcopter flight model as described in section II.

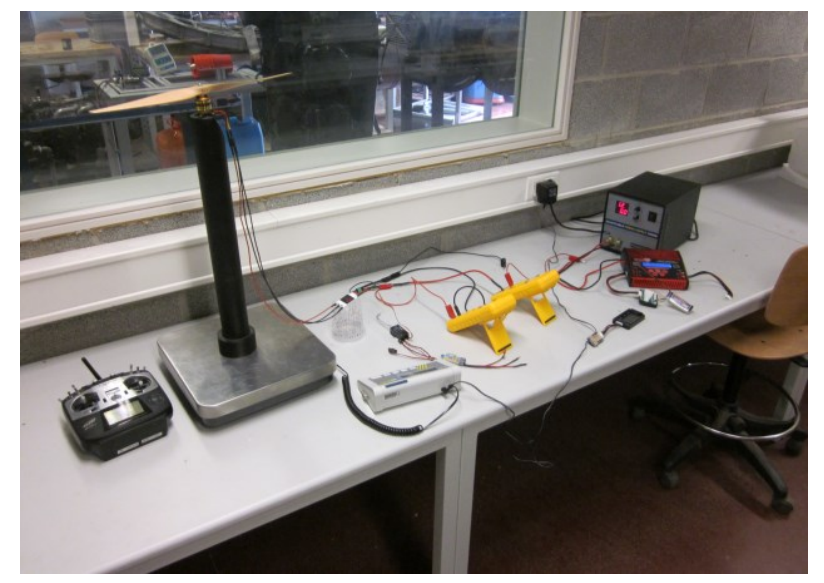

Figure 6. The motor-propeller test setup

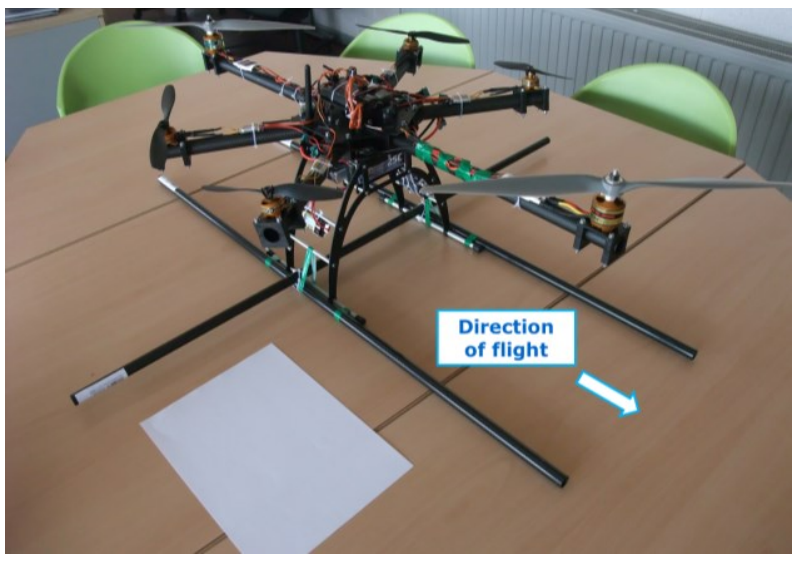

Figure 7. The Demonstrator UAV

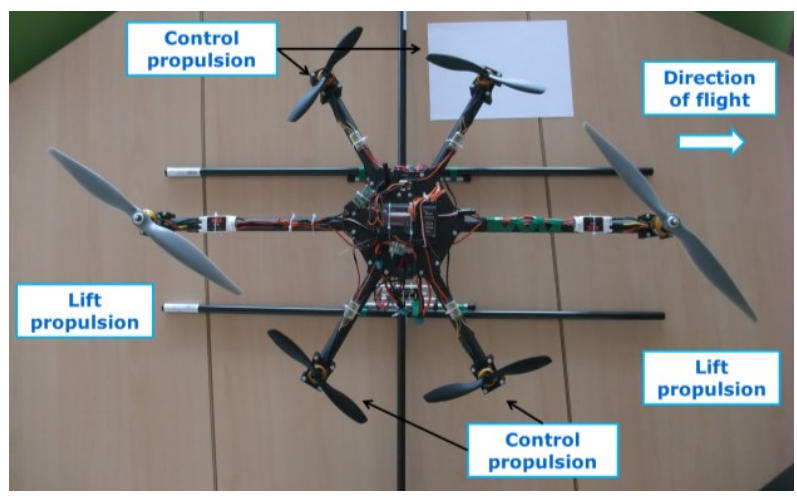

Figure 8. The Demonstrator UAV (top view)

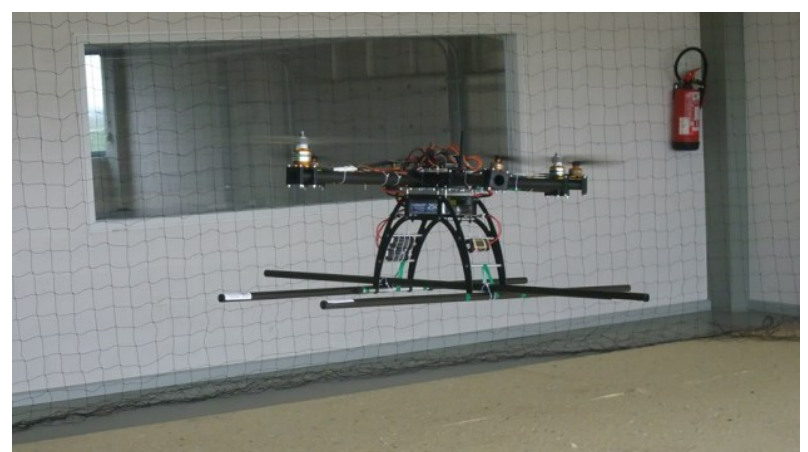

Figure 9. The Demonstrator UAV in flight

Several successful flight tests have been performed (Fig. 9), during which the PIDs of the ArduPilot-Mega have been tuned and estimated endurance has been validated. The tuning of the PID was performed experimentally by the Ziegler-Nichols method [20]. Even though the long and narrow design translates into high inertia in pitch direction and small moment arms together with low available control thrust, the response in pitch of the Demonstrator is excellent. The roll and yaw response are as agile as for a standard hexacopter. If the altitude (controlled by varying the overall thrust) is controlled manually, the response is very sensitive (especially in ground effect), but when controlled by the autopilot it performs well. It is expected that the used of an ultrasonic altitude sensor will improve this further.

\section{CONCLUSION AND FUTURE WORK}

This paper reports on the development of a new multicopter configuration, namely a compound hexacopter. The novel configuration has been conceived as part of a doctoral research in which an unmanned rotary UAV is designed, constructed and flight tested for inspecting fruit orchards and vineyards, more specifically, for estimating harvest yield. The UAV will fly through the orchard autonomously and use side-ward looking cameras to estimate the harvest yield.

By splitting the lift and control propulsion, a compound hexacopter UAV has been designed that combines two large lift propellers and four small control propellers in a rectangularly shaped configuration that is specifically suited for flight through narrow corridors. The configuration easily allows scaling the design that can be used for both outdoor and indoor applications. The compound configuration achieves high agility and long endurance despite limited width. Compared to a standard multicopter (quadcopter) with the same width, payload and battery capacity, the endurance of the compound hexacopter is up to $60 \%$ higher mainly from having a lower disk loading than the standard quadcopter.

Based on the compound hexacopter configuration a small-scaled prototype, named the Demonstrator, has been designed, constructed and flight tested specifically for testing the new configuration without the harvest yield estimation payload. The initial flight tests have shown that the Demonstrator is agile, stable and very responsive to control inputs. The Demonstrator serves as a first step in developing a large-scale multicopter able to autonomously navigate in between tree rows in outdoor conditions for an extended flight time with the application of harvest yield estimation. 
In the future, the Demonstrator will serve as the basis for developing a compound hexacopter flight model (including lift propeller effects) and a robust advanced controller. The controller will be able to fuse the data from the various mission sensors and forward camera image processing hardware while adhering to the mission requirements for flying in a narrow corridor by using the iTasc methodology. iTasc describes the UAV's mission task as a set of constraints, combined with one or more objective functions that the controller solves [21]. In addition, the Demonstrator will be used to acquire structural and in-flight performance data in order to develop a new optimized prototype with high-end components able to carry the new controller and mission sensors.

\section{REFERENCES}

[1] R. Austin, "Unmanned Aircraft Systems: UAVS Design, Development and Deployment”, 2010, Wiley, p. 253-279

[2] M. Luccio, "Remote Sensing by Remote Control: UAVs become invaluable assets", Geospatial Exploration Products BAE Systems, 2009, http://www.geospatialexploitationproducts.com

[3] Andriluka, Schnitzspan, Meyer, Kohlbrecher, Petersen, von Stryk, Roth, and Schiele 2010, "Vision based victim detection from unmanned aerial vehicles", Intelligent Robots and Systems (IROS) conference proceedings, 2010, Taipei, p 1740-1747

[4] J. Vantilt, B. Theys, J. Verbeke, T. Goedemé and J. De Schutter, "Collision Avoidance of an Indoor Quadrotor Helicopter Using Outboard Sensors", 2013, Master thesis, Dept. of Mech. Eng., KU Leuven

[5] J. Verbeke, "Possible applications of a rotary UAS for inspection of orchards", 2012, Doctoral research literature study, Dept. of Mech. Eng., KU Leuven

[6] P.J. Zarco-Tejada, V. González-Dugo and J.A.J. Berni, "Fluorescence, temperature and narrow-band indices acquired from a UAV platform for water stress detection using a micro-hyperspectral imager and a thermal camera", Remote Sensing of Environment, Vol. 117, 2012, p. $322-337$
[7] L. Suárez, P.J. Zarco-Tejada, V. González-Dugo, J.A.J. Berni, R Sagardoy, F. Morales and E. Fereres, "Detecting water stress effects on fruit quality in orchards with time-series PRI airborne imagery", Remote Sensing of Environment, Vol. 114, 2010, p. 286-298

[8] P. Binard, World Apple and Pear Association WAPA, "European apple outlook 2012", Prognosfruit conference, 2012, Toulouse http://www.prognosfruit.eu

[9] A.B.M. Solomakhin, "Mechanical flower thinning improves the fruit quality of apples", 2012, Journal of the Science of Food and Agriculture, Volume 90, no. 5, p. 735-741

[10] A-K Mahlein, "Detection, identification, and quantification of fungal diseases of sugar beet leaves using imaging and non-imaging hyperspectral techniques", 2010, Doctoral thesis, Rheinischen Friedrich-Wilhelms-Universität, Bonn

[11] R.W. Prouty, "Helicopter Performance, Stability and Control”, 2003, Krieger publishing Company, p. 1-10

[12] Microdrones, "MD4-1000", accessed 1 February 2014, http://www.microdrones.com/

[13] E-volo, accessed 1 February 2014, http://www.e-volo.com/

[14] Microdrones, "MD4-3000", accessed 1 Sept. 2013 , http://www.microdrones.com/

[15] RC Groups forum thread, "H-shaped quad", accessed 1 February 2014, http://www.rcgroups.com/forums/showthread.php?t=1682126

[16] San Jose State University, "Heavy Lift Quadcopter", USA, accessed 1 Februari 2014, student project, http://www.incrediblehlq.com/

[17] D. Hulens and M. Vandersteegen, "UAV autonoom laten vliegen in een boomgaard", 2012, Master thesis, Dept of Industr. Eng., College University Lessius

[18] R.W. Prouty, "Helicopter Performance, Stability and Control", 2003, Krieger publishing Company, p. 1-10

[19] DIY Drones, "ArduPilot-Mega”, accessed 1 February 2014, https://code.google.com/p/arducopter/

[20] J.G. Ziegler and N.B. Nichols, "Optimum Settings for Automatic Controllers", 1942, Rochester N.Y.

[21] J. De Schutter, T. De Laet, J. Rutgeerts, W. Decré, R. Smits, E. Aertbeliën, K. Claes and H. Bruyninckx, "Constraint-based task specification and estimation for sensor-based robot systems in the presence of geometric uncertainty", 2007, International Journal of Robotics Research, Vol 25, no. 5, p. 433-455

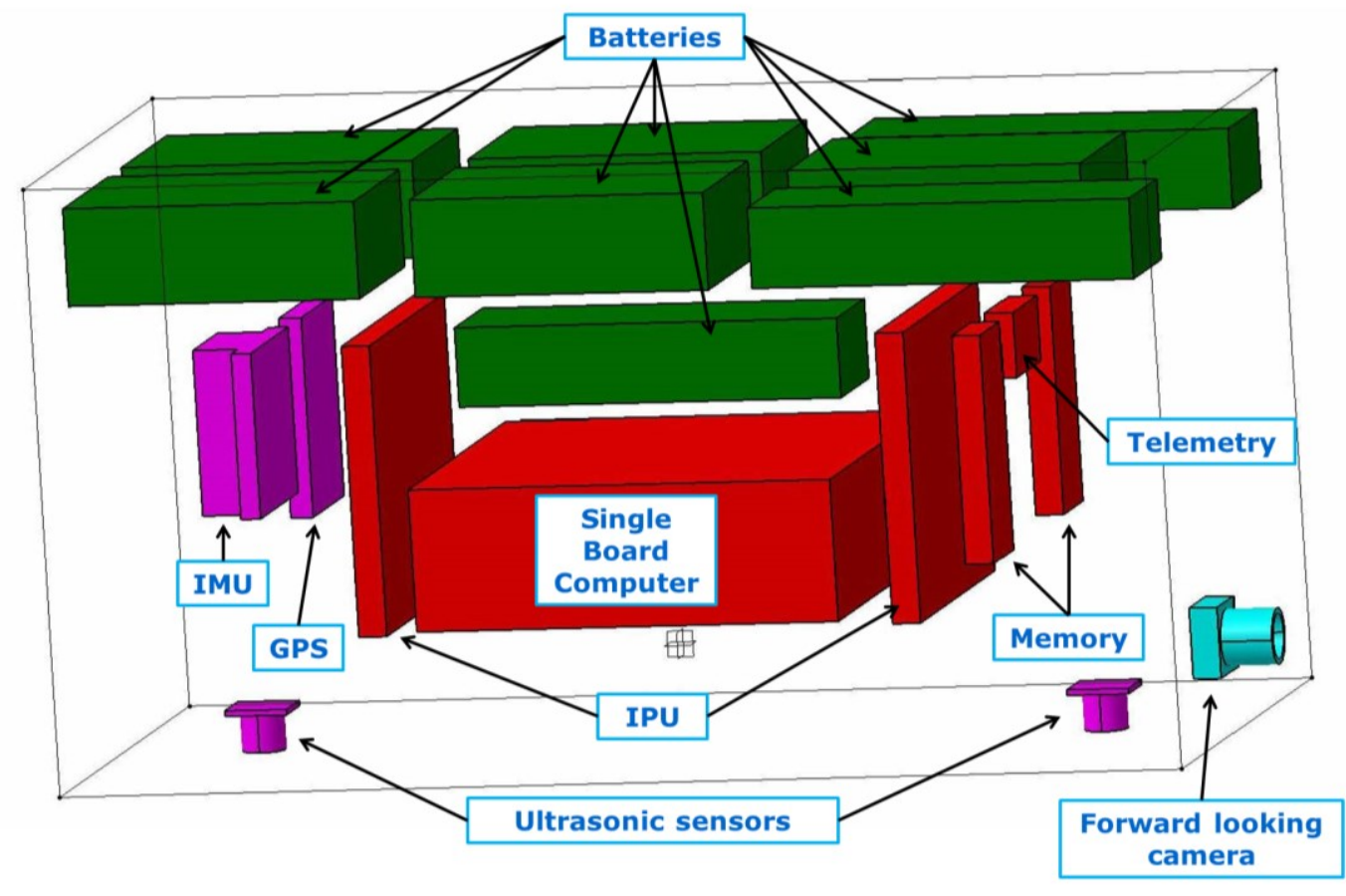

Figure 10. The payloadbox of the compound UAV 


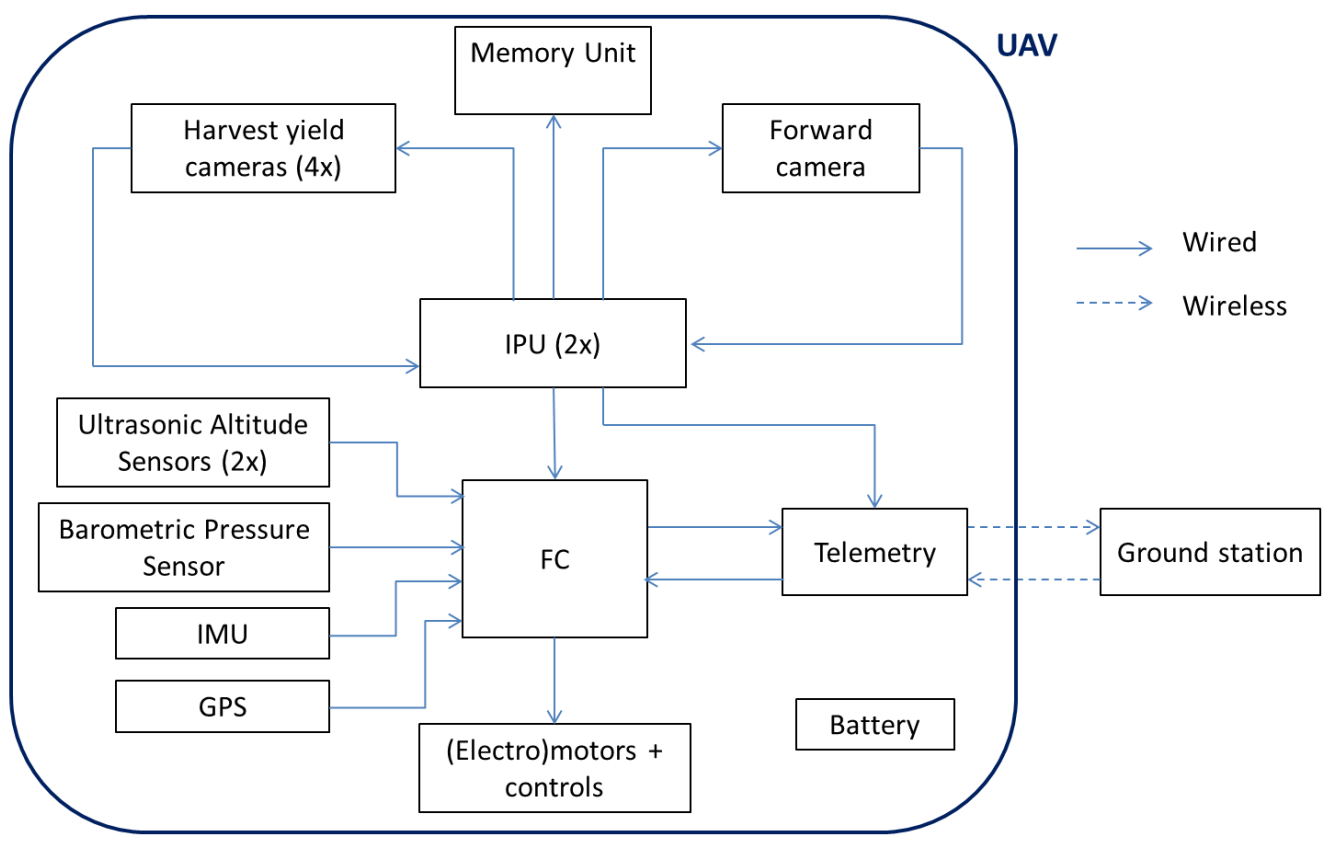

IMU = Inertial Measurement Unit

$\mathrm{FC}=$ Flight Controller

$\mathrm{IPU}=$ Image Processing Unit

GPS = Global Positioning System

Figure 11. The functional diagram of the harvest yield estimation compound UAV

TABLE III. THE COMPONENT LIST OF THE COMPOUND CONCEPTUAL DESIGN

\begin{tabular}{|c|c|c|c|c|c|}
\hline Components & Type & Manufacturer & $\begin{array}{c}\text { Mass } \\
{[\mathrm{g}]}\end{array}$ & Quantity & $\begin{array}{c}\text { Total Weight } \\
{[\mathrm{g}]}\end{array}$ \\
\hline Fruit inspection camera & Guppy F-038 & Allied Vision Tec & 50 & 4 & 200 \\
\hline Lens & MVL4WA & Thorlabs & 70 & 4 & 280 \\
\hline Memory & Memory stick 64GB & Not yet selected & 20 & 2 & 40 \\
\hline Filter & FL05532-10 & Thorlabs & 27 & 4 & 108 \\
\hline Camera gimbal & Custom carbon & Not yet selected & 71 & 1 & 71 \\
\hline Forward camera & FlyCamOne ECO V2 & FlyCamOne & 30 & 1 & 30 \\
\hline Altimeter & MaxSonar-EZ & Maxbotix & 4 & 2 & 8 \\
\hline GPS & AsterX-m & Septentrio & 47 & 1 & 47 \\
\hline IMU & Mti Dev. Kit & Xsens & 50 & 1 & 50 \\
\hline Pressure sensor & MPX4115A & Freescale & 4 & 1 & 4 \\
\hline Telemetry & GS1500M & GainSpan & 100 & 1 & 100 \\
\hline SBC & Not yet selected & Not yet selected & 100 & 1 & 100 \\
\hline IPU & Pandaboard & Pandaboard.org & 74 & 2 & 148 \\
\hline Battery lift & 25C 4S 5400mAh & Thunderpower & 480 & 5 & 2,400 \\
\hline Battery control & 25C 3S 5000mAh & Thunderpower & 357 & 3 & 1,071 \\
\hline Electromotor lift & 2820/14 Gold Line & AXI & 151 & 2 & 302 \\
\hline Electromotor control & 2208/34 Gold line & AXI & 45 & 4 & 180 \\
\hline Propeller lift & $30 \times 9$ & Not yet selected & 150 & 2 & 300 \\
\hline Propeller control & $8 \times 4.7 \mathrm{SF}$ & APC & 8 & 4 & 32 \\
\hline ESC lift & Advance 40 Pro OPTO & Jeti & 35 & 2 & 70 \\
\hline ESC control & Advance Pro 8A & Jeti & 6 & 4 & 24 \\
\hline Structure & Carbon & Not yet selected & 1200 & 1 & 1,200 \\
\hline Total Payload & & & & & 699 \\
\hline Total Computer hardware & & & & & 487 \\
\hline Total propulsion & & & & & 908 \\
\hline Total batteries & & & & & 3,471 \\
\hline Total structure & & & & & 1,200 \\
\hline Total UAV & & & & & 6,765 \\
\hline
\end{tabular}


TABLE IV. THE COMPONENT LIST OF THE STANDARD QUADCOPTER USED FOR COMPARISON WITH THE COMPOUND DESIGN

\begin{tabular}{|c|c|c|c|c|c|}
\hline Components & Type & Manufacturer & $\begin{array}{c}\text { Mass } \\
{[\mathrm{g}]}\end{array}$ & Quantity & $\begin{array}{c}\text { Total Weight } \\
{[\mathrm{g}]}\end{array}$ \\
\hline Fruit inspection camera & Guppy F-038 & Allied Vision Tec & 50 & 4 & 200 \\
\hline Lens & MVL4WA & Thorlabs & 70 & 4 & 280 \\
\hline Memory & Memory stick 64GB & Not yet selected & 20 & 2 & 40 \\
\hline Filter & FL05532-10 & Thorlabs & 27 & 4 & 108 \\
\hline Camera gimbal & Custom carbon & Not yet selected & 71 & 1 & 71 \\
\hline Forward camera & FlyCamOne ECO V2 & FlyCamOne & 30 & 1 & 30 \\
\hline Altimeter & MaxSonar-EZ & Maxbotix & 4 & 2 & 8 \\
\hline GPS & AsterX-m & Septentrio & 47 & 1 & 47 \\
\hline IMU & Mti Dev. Kit & Xsens & 50 & 1 & 50 \\
\hline Pressure sensor & MPX4115A & Freescale & 4 & 1 & 4 \\
\hline Telemetry & GS1500M & GainSpan & 100 & 1 & 100 \\
\hline SBC & Not yet selected & Not yet selected & 100 & 1 & 100 \\
\hline IPU & Pandaboard & Pandaboard.org & 74 & 2 & 148 \\
\hline Battery & 25C 5S 5400mAh & Thunderpower & 589 & 6 & 3,534 \\
\hline Electromotor & 2826/12 Gold Line & AXI & 181 & 4 & 724 \\
\hline Propeller & $12 \times 4.7 \mathrm{SF}$ & APC & 20 & 4 & 80 \\
\hline ESC & Advance 40 Pro OPTO & Jeti & 35 & 4 & 140 \\
\hline Structure & Carbon & Not yet selected & 1000 & 1 & 1,000 \\
\hline Total Payload & & & & & 699 \\
\hline Total Computer hardware & & & & & 487 \\
\hline Total propulsion & & & & & 944 \\
\hline Total batteries & & & & & 3,534 \\
\hline Total structure & & & & & 1,000 \\
\hline Total UAV & & & & & $6,6,664$ \\
\hline
\end{tabular}

